\title{
Spontaneous polarization and locomotion of an active particle with surface-mobile enzymes
}

\author{
Marco De Corato ${ }^{*}$ \\ Institute for Bioengineering of Catalonia (IBEC), The Barcelona Institute of Science and Technology (BIST), \\ Baldiri Reixac 10-12, 08028 Barcelona, Spain \\ Ignacio Pagonabarraga ${ }^{\circ}$ \\ Departament de Física de la Matèria Condensada, Universitat de Barcelona, C. Martí Franquès 1, \\ 08028 Barcelona, Spain; \\ University of Barcelona Institute of Complex Systems (UBICS), Universitat de Barcelona, \\ 08028 Barcelona, Spain; \\ and CECAM, Centre Européen de Calcul Atomique et Moléculaire, École Polytechnique Fédérale de \\ Lasuanne (EPFL), Batochime, 1015 Lausanne, Switzerland
}

Loai K. E. A. Abdelmohsen

Department of Biomedical Engineering, Department of Chemical Engineering and Chemistry, Institute for Complex Molecular Systems Eindhoven University of Technology, P. O. Box 513, Eindhoven MB 5600, The Netherlands

Samuel Sánchez $\odot$

Institute for Bioengineering of Catalonia (IBEC), The Barcelona Institute of Science and Technology (BIST), Baldiri Reixac 10-12, 08028 Barcelona, Spain;

Institució Catalana de Recerca i Estudis Avançats (ICREA), 08010 Barcelona, Spain; and School of Materials Science and Engineering, Harbin Institute of Technology Shenzhen, 518055 China

\author{
Marino Arroyo ${ }^{\dagger}$ \\ Universitat Politècnica de Catalunya-BarcelonaTech, 08034 Barcelona, Spain; \\ Institute for Bioengineering of Catalonia (IBEC), The Barcelona Institute of Science and Technology (BIST), \\ Baldiri Reixac 10-12, 08028 Barcelona, Spain \\ and Centre Internacional de Mètodes Numèrics en Enginyeria (CIMNE), 08034 Barcelona, Spain
}

(Received 31 August 2020; accepted 20 November 2020;

published 17 December 2020)

\begin{abstract}
We examine a mechanism of locomotion of active particles whose surface is uniformly coated with mobile enzymes. The enzymes catalyze a reaction that drives phoretic flows but their homogeneous distribution forbids locomotion by symmetry. We find that the ability of the enzymes to migrate over the surface combined with self-phoresis can lead to a spontaneous symmetry-breaking instability whereby the homogeneous distribution of enzymes polarizes and the particle propels. The instability is driven by the advection of enzymes by the phoretic flows and occurs above a critical Péclet number. The transition to polarized motile states occurs via a supercritical or subcritical pitchfork bifurcations, the latter of which enables hysteresis and coexistence of uniform and polarized states.
\end{abstract}

\footnotetext{
*mdecorato@ibecbarcelona.eu

†'marino.arroyo@upc.edu
} 
DOI: 10.1103/PhysRevFluids.5.122001

Introduction. Eukaryotic cells and bacteria use chemical energy to move in various environments. Mimicking living cells and given the availability of chemical energy in the environment, artificial colloidal particles can be designed to self-propel through surface chemical reactions [1,2]. Besides serving as a model system to explore collective nonequilibrium phenomena [3], several technological applications have been envisaged for these active particles: from biomedical [4-6] to environmental remediation [7]. To achieve self-propulsion, different mechanism have been proposed such as diffusiophoresis [8], thermophoresis [9,10], momentum exchange [11], release of ions [12], and liquid-liquid phase separation [13].

Regardless of the mechanism, a requirement of self-propulsion is symmetry breaking. This has been achieved by hard-wiring onto the material particle an asymmetric shape [14-17] or an asymmetric catalytic reaction rate [18], both of which pose manufacturing challenges particularly at smaller scales. While built-in asymmetry is intrinsic to flagellates and other microorganisms, animal cells have the ability to dynamically develop self-polarization of their active cytoskeleton, thereby switching between quiescent and motile states [19-22]. If polarization is not built-in but is instead an emergent response that can be triggered on demand, then this may lead to tunable, adaptable, and more easily produced self-propelled particles.

Here we propose a self-propulsion strategy based on enzyme catalysis [23-27] that does not require a built-in asymmetric catalytic reaction. Rather than using a fixed catalyst, we consider chemically active colloids coated with mobile enzymes. To maximize the entropy, these mobile molecules will tend to homogeneously distribute on the particle's surface. We hypothesize that spontaneous polarization and propulsion may arise as an advective instability driven by the interplay between the surface mobility of enzymes, a chemical reaction and self-diffusiophoresis, as sketched in Fig. 1. The mechanism studied here bears some similarity to the spontaneous symmetry-breaking instability observed for isotropic catalytic particles, which is driven by the advection of the reaction product [28-33]. However, here it is the advection along the surface of the enzymes that catalyze the reaction which leads to the spontaneous symmetry-breaking instability.

Governing equations. To examine this idea, we consider a spherical particle of radius $R$ that is covered by enzymes and is suspended in a liquid, see Fig. 1(a). The enzymes are constrained to the surface of the particle but are free to move laterally. Migration of macromolecules over a surface occurs frequently in nature, a notable example being membrane-associated proteins [34-36]. We assume that the enzymes are much smaller than the particle, which is the case for micron-sized particles and nanometer-sized enzymes. We thus describe the enzymes through their time-dependent surface number density, $\Gamma$. We neglect thermal fluctuations and the deformation of the surface, which may be important for large and flaccid membranes [37].

In the presence of their substrate, the enzymes catalyze a reaction that releases a product species. We assume that the reaction rate is proportional to the local number density of enzymes $k_{\mathrm{cat}} \Gamma$, with $k_{\text {cat }}$ the turnover rate of the enzyme. This simple choice is pertinent when the substrate concentration is much larger than the Michaelis-Menten constant of the enzyme. The product is released from the surface of the particle and then quickly diffuses in the bulk so that advection is negligible [38]. It follows that its distribution relaxes instantaneously to steady state after any change of the enzyme distribution. Under these assumptions, the balance of the number density of the product, $c$, satisfies the Laplace equation,

$$
D \nabla^{2} c=0
$$

with $D$ the diffusion coefficient. At $r \rightarrow \infty$ the concentration of the product is kept constant at $c=0$ and the surface flux of product at $r=R$ is proportional to the reaction rate $-D \nabla c \cdot \boldsymbol{n}=k_{\mathrm{cat}} \Gamma$, with $\boldsymbol{n}$ the unit outer normal vector to the surface of the sphere. The main difference with previous models considering chemically active colloids is that the enzymes are mobile and therefore $\Gamma$ can change in space and time. 


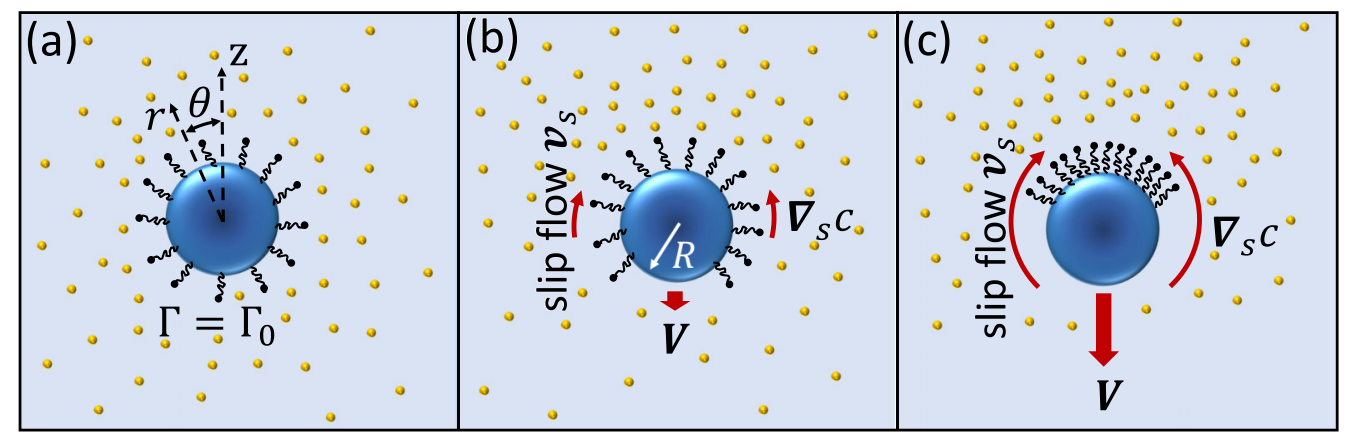

FIG. 1. Self-propulsion mechanism for a colloidal particle (blue) covered by mobile enzymes (black) in a suspension of molecules catalyzed by the enzymes (yellow). (a) In an unpolarized state, the laterally mobile enzymes are homogeneously distributed on the surface to maximize entropy and $\Gamma=\Gamma_{0}$. (b) A perturbation of the distribution of enzymes leads to an inhomogeneous reaction rate, which generates an imbalance of product on the two sides of the particle. The gradient of product results in phoretic flows that transport more enzymes. On a perturbation the system can evolve toward (c), a self-sustained polarization of the enzyme distribution, or go back to (a), a homogeneous state.

The reaction product interacts with the surface of the particle through a short-range potential and its gradients along the particle's surface generate lateral gradients of pressure within a thin boundary layer next to the surface [38,39]. The size of the boundary layer depends on the details of the product-surface interactions but its thickness is usually in the order of a few nanometers [8]. The pressure gradient inside the boundary layer is balanced by the viscous shear stress resulting in an apparent slip velocity that develops over a few nanometers from the surface. This mechanism is effectively described through a slip velocity at the surface of the particle that is proportional to the surface gradient of product and given by $\boldsymbol{v}_{s}=b \nabla_{s} c$, where $b$ is the phoretic mobility coefficient depending on the details of the product-surface interactions [39], which we assume to be constant. Attractive interactions lead to a negative $b$, while repulsive interactions lead to a positive $b$. Finally, for vanishing fluid inertia, the particle velocity is $\boldsymbol{V}=-\left\langle\boldsymbol{v}_{s}\right\rangle$, where \langle\rangle denotes the average over the surface [39].

The enzymes are transported along the surface by diffusion and by the local slip velocity. In a reference frame attached to the center of the particle, their distribution satisfies

$$
\frac{\partial \Gamma}{\partial t}=-\nabla_{s} \cdot\left(\boldsymbol{J}_{s}+f \boldsymbol{v}_{s} \Gamma\right)
$$

where $\boldsymbol{J}_{s}$ is the diffusive flux of enzymes and $f \boldsymbol{v}_{s} \Gamma$ is flux of enzymes driven by the local slip flow. Since the phoretic velocity goes from zero at the surface to $\boldsymbol{v}_{s}$ over a few nanometers, we assume that the enzymes are advected by an effective velocity, $f \boldsymbol{v}_{s}$, that is a fraction of the slip velocity $\boldsymbol{v}_{s}$ observed far from the particle surface. The dimensionless coefficient $f$ takes values between zero and 1 . We are unaware of studies that consider the transport of surface-bound enzymes or proteins due to diffusiophoretic slip flows, which would allow an accurate estimation of the coefficient $f$.

The diffusive flux of enzymes is proportional to the gradient of their chemical potential, $\mu$, along the surface

$$
J_{s}=-D_{s} \Gamma \nabla_{s} \mu / k_{B} T,
$$

with $D_{s}$ the surface diffusion coefficient of the enzymes, $k_{B}$ the Boltzmann constant, and $T$ the absolute temperature. The diffusion of enzymes and proteins along membranes is usually much slower than that of small molecules in a liquid $D_{s} \ll D[40]$, thus neglecting the advective transport of $c$ in the bulk but considering it on the surface is justified. We assume that the chemical potential that drives the diffusive flux derives from the Flory-Huggins free energy, 
$\mu=k_{B} T \log \Gamma /\left(\Gamma_{\infty}-\Gamma\right)+\chi \Gamma-\Lambda \nabla_{s}^{2} \Gamma[36,41,42]$. The chemical potential includes the entropy of mixing, a maximum number density, $\Gamma_{\infty}$, and enzyme-enzyme interactions through $\chi$ and $\Lambda$. A negative $\chi$ corresponds to attractive enzyme-enzyme interactions, which can result in phase separation with coexisting regions of high and low surface concentration of enzymes [43]. Enzymes and proteins suspended in solution often aggregate above a threshold concentration, which suggests some degree of attraction even when they lie on a surface. The last term of the chemical potential accounts for nonlocal interactions between the enzymes. By penalizing lateral gradients of enzymes, it regularizes the boundaries between regions of high and low concentration of enzymes and it is mathematically required when $\chi<0$ [44]. Finally, we assume that the interactions between the enzymes and the product are negligible compared to those between the product and the surface. It follows that $b$ does not depend on $\Gamma$.

Dimensionless equations. We make Eqs. (1) and (2) dimensionless using $R$ as characteristic length scale, $R^{2} / D_{s}$ as characteristic time, $\Gamma_{\infty}$ as characteristic enzyme area density and $k_{\text {cat }} \Gamma_{\infty} R / D$ as characteristic product number density. By doing so, we find four dimensionless numbers. The Péclet number, $\mathrm{Pe}=f b k_{\mathrm{cat}} R \Gamma_{\infty} / D D_{s}$, expresses the relative importance of advection and diffusion of enzymes over the surface. Since the phoretic mobility coefficient can be positive or negative, $\mathrm{Pe}$ is also signed. The sign of Pe indicates whether the enzymes are advected along or against the surface gradient of product. Drift velocities of tens to hundreds of nanometers per second have been measured in experiments employing enzymes driven along lipid membranes by electrophoretic flows [34] or by shear stresses [45]. By considering a velocity of just $10 \mathrm{~nm} / \mathrm{s}$ and a surface diffusion $D_{s} \approx 1 \mu \mathrm{m}^{2} \mathrm{~s}^{-1}$, results $\mathrm{Pe} \approx 1$ for $R \approx 1 \mu \mathrm{m}$. The dimensionless enzyme-enzyme interaction is given by $\chi^{*}=\chi / k_{B} T \Gamma_{\infty}$. The dimensionless nonlocal enzyme-enzyme interaction parameter is defined as $\Lambda^{*}=\Lambda / k_{B} T R^{2} \Gamma_{\infty}$ and it is always positive. Finally, the mean number density divided by the maximum density $\Gamma_{0}^{*}=\Gamma_{0} / \Gamma_{\infty}$ represents the degree of coverage of the surface and varies between zero and one.

The Laplace equation given by Eq. (1) reads, in dimensionless form,

$$
\nabla^{2} c=0 .
$$

With boundary conditions at $r \rightarrow \infty$ given by $c=0$ and at the surface of the particle $r=1$ is given by $-\nabla c \cdot \boldsymbol{n}=\Gamma$. The dimensionless slip velocity becomes $\boldsymbol{v}_{s}=\frac{b k_{\mathrm{cat}} R \Gamma_{\infty}}{D D_{s}} \nabla_{s} c$. By plugging in the definition of the chemical potential into the Eqs. (2) and (3) and making it dimensionless, we obtain

$$
\frac{\partial \Gamma}{\partial t}=\nabla_{s} \cdot\left[\left(\frac{1}{1-\Gamma}+\chi^{*} \Gamma\right) \nabla_{s} \Gamma-\Lambda^{*} \Gamma \nabla_{s} \nabla_{s}^{2} \Gamma-\operatorname{Pe} \nabla_{s} c \Gamma\right] .
$$

Finally, the dimensionless velocity of the particle becomes $\boldsymbol{V}=-\frac{\mathrm{Pe}}{4 \pi f} \int_{S} \nabla_{s} c d S$.

It is straightforward to show that $\Gamma=\Gamma_{0}^{*}$ and by $c=\Gamma_{0}^{*} / r$ are a solution to the Eqs. (4) and (5), which corresponds to a spherically symmetric distribution of $c$ around the particle. However, this solution can become unstable to infinitesimal fluctuations of the enzyme distribution. We study this phenomenon by performing a linear stability analysis of the homogeneous solution and fully nonlinear numerical simulations of Eqs. (4) and (5).

Linear stability analysis. We consider small axisymmetric perturbations $\Gamma=\Gamma_{0}^{*}+\delta \Gamma$ and $c=\Gamma_{0}^{*} / r+\delta c$ about the homogeneous steady state and expand $\delta c$ and $\delta \Gamma$ in Legendre polynomials as $\delta c=\sum_{l=1}^{\infty} \delta c_{l}(0) \exp \left(\lambda_{l} t\right) r^{-l-1} P_{l}(\cos \theta)$ and $\delta \Gamma=\sum_{l=1}^{\infty} \delta \Gamma_{l}(0) \exp \left(\lambda_{l} t\right) P_{l}(\cos \theta)$, with $P_{l}(\cos \theta)$ the Legendre polynomial of degree $l$ and $\theta$ the polar angle. $\delta \Gamma_{l}(0)$ and $\delta c_{l}(0)$ are the initial values of each Legendre mode and $\lambda_{l}$ is their growth rate. If the real part of $\lambda_{l}$ is positive, then any perturbation of the mode $l$ grows exponentially and the homogeneous state is unstable. By plugging the expansions $\Gamma=\Gamma_{0}^{*}+\delta \Gamma$ and $c=\Gamma_{0}^{*} / r+\delta c$ into the governing Eqs. (4) and (5) and keeping only the linear terms, we find a relation between the growth rate $\lambda_{l}$, the mode number $l$ and the dimensionless numbers [46]:

$$
\lambda_{l}=-l(l+1)\left[\frac{1}{1-\Gamma_{0}^{*}}+\chi^{*} \Gamma_{0}^{*}+l(l+1) \Lambda^{*} \Gamma_{0}^{*}\right]+l \Gamma_{0}^{*} \mathrm{Pe} .
$$


The instability condition, $\lambda_{l}>0$, can be rewritten as a condition for the Péclet number,

$$
\operatorname{Pe} \Gamma_{0}^{*}>(l+1)\left[\left(1-\Gamma_{0}^{*}\right)^{-1}+\Gamma_{0}^{*} \chi^{*}+l(l+1) \Gamma_{0}^{*} \Lambda^{*}\right]
$$

In the case of weakly attractive or repulsive enzyme-enzyme interactions, $\chi^{*} \geqslant-\left[\left(1-\Gamma_{0}^{*}\right) \Gamma_{0}^{*}\right]^{-1}-$ $2 \Lambda^{*}$ the equilibrium distribution of enzymes is homogeneous. In these cases, the right-hand side of the inequality above is a monotonically increasing function of $l$ and the first unstable mode is the dipole, $l=1$. Thus, we define a critical Péclet number,

$$
\mathrm{Pe}_{\mathrm{crit}}=\frac{2}{\left(1-\Gamma_{0}^{*}\right) \Gamma_{0}^{*}}+2 \chi^{*}+4 \Lambda^{*},
$$

that discriminates homogeneous states that are stable from those that are unstable. Any homogeneous state with $\mathrm{Pe}>\mathrm{Pe}_{\text {crit }}$ is unstable to infinitesimal perturbations and spontaneously polarizes. In this case, the phoretic flows advect more enzymes along the gradient of concentration and reinforce an initial perturbation as depicted in Fig. 1(b). Equation (8) shows that attractive enzymeenzyme interactions, $\chi^{*}<0$, promote the instability by favoring their aggregation, thus reducing $\mathrm{Pe}_{\text {crit }}$. Conversely, $\Lambda^{*}$ penalizes the formation of inhomogeneous distributions, thus hindering the spontaneous polarization. In the case of no enzyme-enzyme interactions $\chi^{*}=\Lambda^{*}=0$, and of a dilute surface $\Gamma_{0}^{*} \approx 0$, the instability condition can be rewritten simply as $f k_{\text {cat }} k_{B} T \Gamma_{0} R / D D_{s}>2$. Note that, in the absence of surface saturation or enzyme-enzyme repulsion, there is no mechanisms that prevents an infinite local accumulation of enzymes. In the case of strong enzyme-enzyme attractive interactions $\chi^{*}<-\left[\left(1-\Gamma_{0}^{*}\right) \Gamma_{0}^{*}\right]^{-1}-2 \Lambda^{*}$, the homogeneous distribution of enzymes phase separates at equilibrium to form a enzyme-concentrated phase coexisting with an enzymedepleted one. However, the homogeneous distribution can be stabilized by the phoretic flows with a negative Péclet number. This occurs if $\mathrm{Pe}$ is negative and its magnitude is sufficiently large so that the growth rate, given by Eq. (6), is negative for any mode $l$. In this case, the slip flow direction is reversed with respect to that shown in Fig. 1(b) and acts against the gradients of concentration of enzymes, therefore stabilizing the homogeneous distribution versus the attractive interactions between the enzymes.

Enzyme distribution and locomotion at steady state. To examine the distribution of enzymes and the particle velocity beyond the linear stability analysis, we resort to time-dependent numerical simulations [46]. We assume an axisymmetric solution and we expand the bulk and surface concentrations in Legendre modes, $c=\sum_{l=1}^{\infty} c_{l}(t) r^{-l-1} P_{l}(\cos \theta)$ and $\Gamma=\sum_{l=1}^{\infty} \Gamma_{l}(t) P_{l}(\cos \theta)$, and solve for the time-dependent coefficients $c_{l}(t)$ and $\Gamma_{l}(t)$. More details about the numerical method used to solve Eqs. (4) and (5) are found in Ref. [46]. By plugging in the expansion of $\Gamma$ and of $c$ into the expression of the dimensionless velocity, we find that the velocity of the particle is directly related to the dipolar mode, $\Gamma_{1}(t)$, as $\boldsymbol{V}=-\frac{\mathrm{Pe}}{3 f} \Gamma_{1}(t) \hat{z}$, with $\hat{z}$ the unit vector along the $z$ axis [46].

In Fig. 2(a), we show the steady-state velocity of an active particle as a function of the Péclet number for $\Gamma_{0}^{*}=0.5$, no interactions between enzymes $\chi^{*}=0$ and $\Lambda^{*}=0$. As shown in the insets of Fig. 2(a), at small Péclet numbers the homogeneous distribution of enzymes is stable and the particle does not move. The velocity undergoes a supercritical pitchfork bifurcation at $\mathrm{Pe}=\mathrm{Pe}_{\text {crit }}$ whereby the quiescent solution becomes unstable, the spherical symmetry breaks and two polarized steady states become stable. In fact, polarization can emerge in any direction but, without loss of generality, our parametrization of the solutions describes only two of these directions. The critical Péclet number matches that predicted by the linear stability analysis. At $\mathrm{Pe}>\mathrm{Pe}_{\text {crit }}$, the spherical symmetry breaks, resulting in an asymmetry not only of the enzymes but also of the product, Fig. 2(b).

The velocity streamlines are shown in Fig. 2(b) in the comoving frame. Since the flow field is generated by a surface slip velocity, we can use the squirmer model $[47,48]$ to rationalize it. We find that, the particle does not exert a force dipole to the fluid and behaves as a neutral squirmer [49]. Therefore, the far field velocity field decays as $r^{-3}$ in the far field. This finding has implications for 

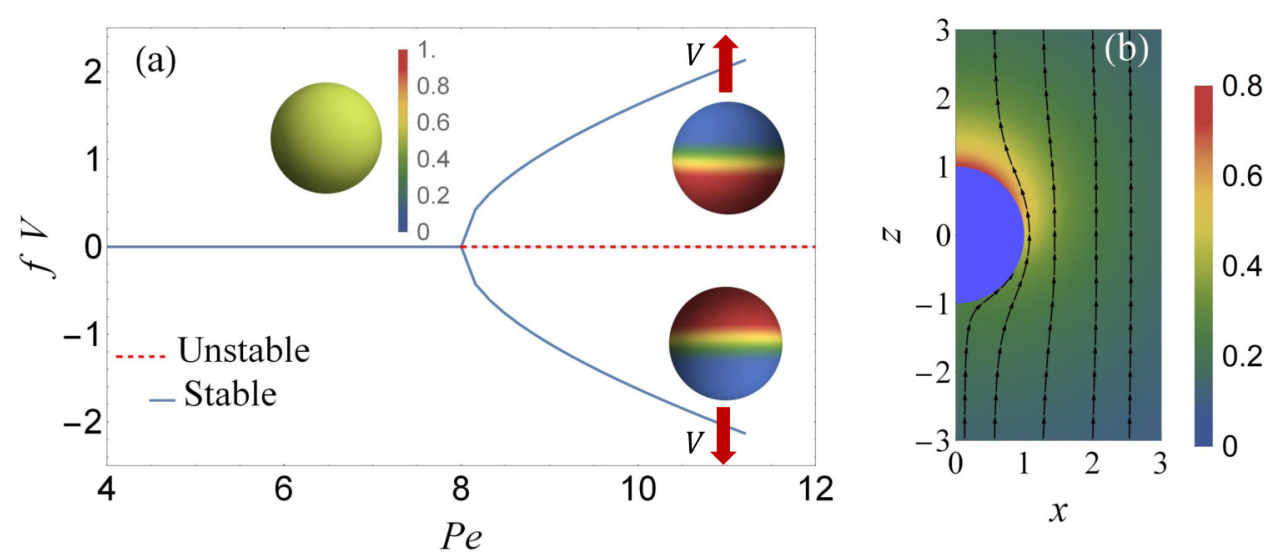

FIG. 2. Spontaneous polarization and locomotion: (a) The dimensionless velocity of the particle as a function of Pe for $\Gamma_{0}^{*}=0.5$ and no enzyme-enzyme interaction $\chi^{*}=0$ and $\Lambda^{*}=0$. The insets show the dimensionless enzyme distribution in homogeneous and polarized states. (b) The dimensionless concentration of the product around the particle and the streamlines corresponding to $\mathrm{Pe}=11$.

the collective motion of multiple particles as hydrodynamic interactions might decay faster than the particle-particle phoretic interactions [50-56].

By reducing $\Gamma_{0}^{*}$ from $\Gamma_{0}^{*}=0.5$ to $\Gamma_{0}^{*}=0.1$, the pitchfork bifurcation occurring at $\mathrm{Pe}=\mathrm{Pe}_{\text {crit }}$ changes from supercritical to subcritical. This is depicted in Fig. 3, where we plot the velocity of the particle for $\Gamma_{0}^{*}=0.1$. In contrast to what we found for $\Gamma_{0}^{*}=0.5$, Fig. 3 shows that stable polarized and stable homogeneous solutions coexist for a range of Péclet numbers. As a consequence, a particle with an homogeneous distribution of enzymes suddenly jumps to a polarized state with a finite velocity, once $\mathrm{Pe}>\mathrm{Pe}_{\text {crit }}$. By increasing and then decreasing the Péclet number, the distribution of enzymes undergoes a hysteresis loop: a polarized state emerges for $\mathrm{Pe}>\mathrm{Pe}_{\text {crit }}$ and disappears for $\mathrm{Pe}<\mathrm{Pe}_{\text {coex }}$ with $\mathrm{Pe}_{\text {coex }}<\mathrm{Pe}_{\text {crit }}$. Such hysteresis loop might be observed in experiments where

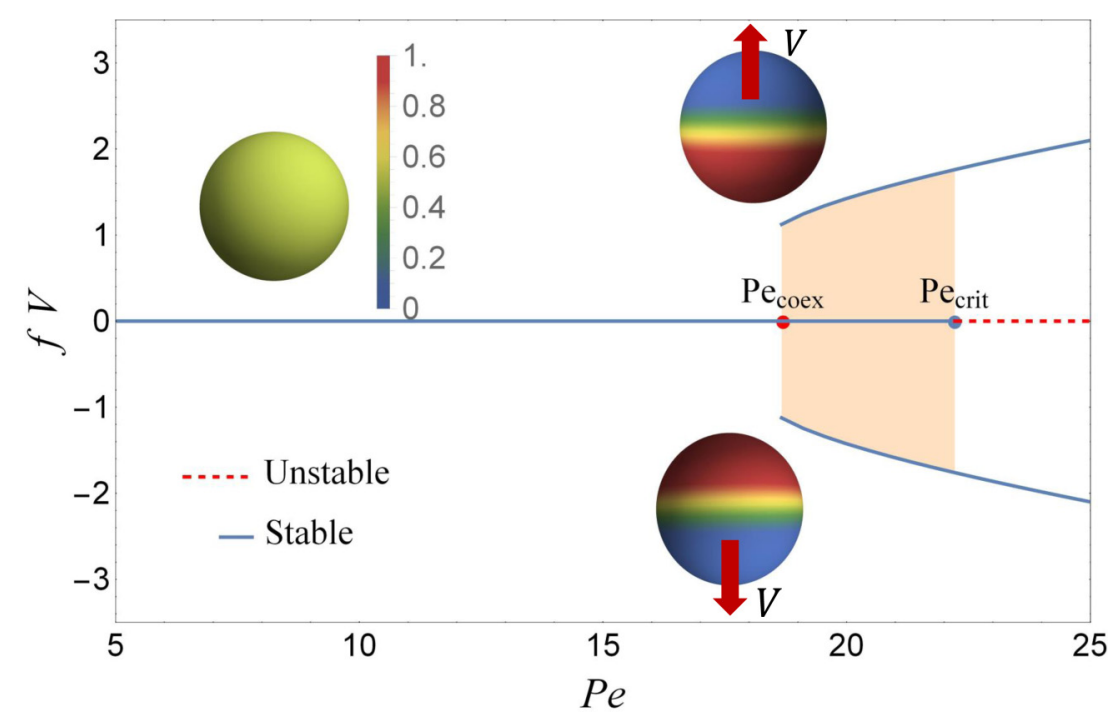

FIG. 3. The dimensionless velocity of the particle as a function of Pe for $\Gamma_{0}^{*}=0.1$ and $\chi^{*}=0$ and $\Lambda^{*}=0$. The shaded area represents the region where stable homogeneous and polarized solutions coexist. 

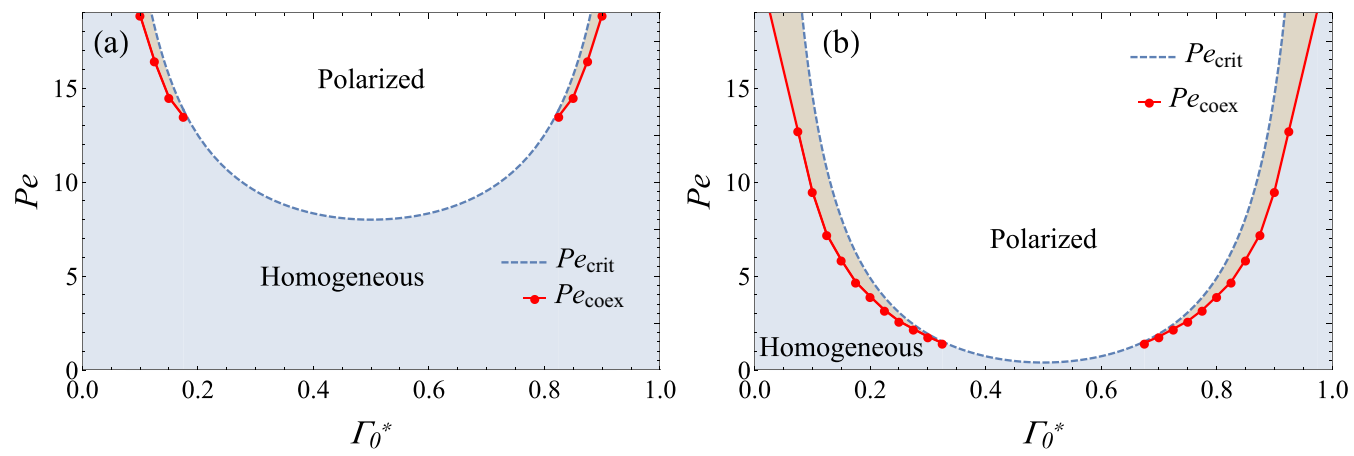

FIG. 4. Phase diagrams of the enzyme distribution in the case (a) no enzyme-enzyme interactions, $\chi^{*}=0$ and $\Lambda^{*}=0$ and (b) attractive enzyme-enzyme interactions, $\chi^{*}=-4$ and $\Lambda^{*}=0.1$. The blue-shaded area denotes regions where the homogeneous state is the only stable solution. In the white region the only stable solution is the polarized state. The orange-shaded region represents parameters for which the polarized and homogeneous solutions coexist. $\mathrm{Pe}_{\text {crit }}$ is given by Eq. (8) while $\mathrm{Pe}_{\text {coex }}$ is computed using numerical simulations [46].

the Péclet number is tuned through the reaction rate or where an external field is used to drive the system from the homogeneous stable branch to the polarized one.

The overall behavior of the active particle is summarized in Fig. 4, where we map the regions where the homogeneous state, the polarized state, or both are stable depending on the average coverage of enzymes $\Gamma_{0}^{*}$ and Pe. We present such diagrams in the cases of no enzyme-enzyme interactions and of attractive enzyme-enzyme interactions. In both cases, polarized solutions can arise at lower Pe for intermediate enzyme coverages. The appearance of a minimum $\mathrm{Pe}_{\text {crit }}$ at $\Gamma_{0}^{*}=0.5$ can be understood as the competition of two mechanisms. By increasing $\Gamma_{0}^{*}$ the reaction rate becomes faster, which results in stronger phoretic flows thus promoting the instability. However, increasing $\Gamma_{0}^{*}$ also leads to a more crowded surface, which hinders the spontaneous polarization. The competition between these two mechanisms leads to a minimum Pe $\mathrm{P}_{\text {crit }}$. For noninteracting enzymes and for $\Gamma_{0}^{*}=0.5$, the critical Pe is about 8, Fig. 4(a), which requires fast reaction kinetics, large phoretic mobility coefficient, large particle size, and/or slow surface diffusion. In addition to these somewhat experimentally controllable knobs, an attractive self-interaction between enzymes of a few $k_{B} T$ can significantly expand the regions of coexistence and reduce $\mathrm{Pe}_{\text {crit }}$, below one for $\Gamma_{0}^{*}=0.5$, Figs. 4(b).

The behavior of an active particle whose surface distribution is phase separated at equilibrium due to attractive enzyme-enzyme interactions is shown in Fig. 5. In this figure, we report the steady-state value of the dipole of the enzyme concentration, $\Gamma_{1}$, as a function of Pe for $\Gamma_{0}^{*}=0.5, \chi^{*}=-6$, and $\Lambda^{*}=0.1$. We find that the two stable polarized branches, with strictly positive and negative $\Gamma_{1}$, extend from positive to negative value of Pe and disappear through a supercritical bifurcation when Pe is smaller than a negative threshold. In the polarized state, the active particle can propel either towards or against the portion of the surface having larger enzyme concentration, depending on the sign of Pe, see Figs. 5(i) and 5(ii). Interestingly, for $\mathrm{Pe}<-3.6$ the stable solution is not given by a homogeneous distribution of enzymes. Instead, for $\mathrm{Pe}<-3.6$, we find that the surface distribution displays higher order modes $\Gamma_{l} \neq 0$ for $l>1$ with the dominant mode being $l=2$, see Fig. 5(iii). In this case, advection penalizes the formation of gradients of enzymes with long wavelength $l=1$. This steady state represents a nonmotile configuration whereby the active particle generates steady flows. Finally, by further decreasing the Péclet number beyond $\mathrm{Pe}<-4.2$, the stable solution becomes the homogeneous distribution of enzymes with no flow, see Fig. 5(iv). These findings agree quantitatively with the prediction of the linear stability analysis, given by 


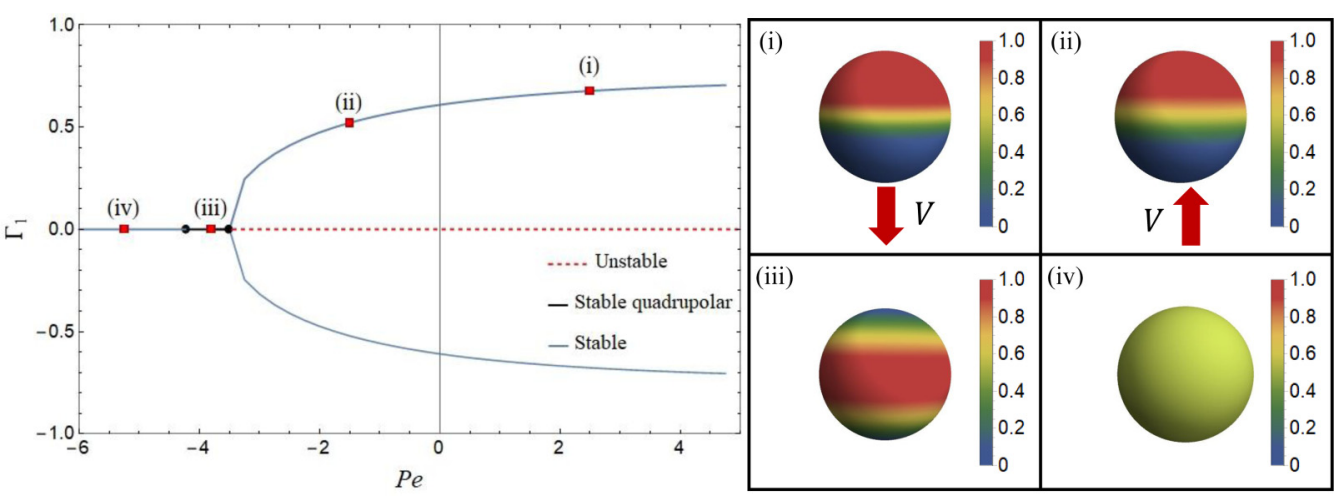

FIG. 5. Dipolar component of the enzyme distribution, $\Gamma_{1}$, in the case of $\Gamma_{0}^{*}=0.5, \chi^{*}=-6$ and $\Lambda^{*}=0.1$. The panels (i)-(iv) display the enzyme distribution corresponding to the same symbols in the left figure.

Eq. (6), which predicts that only the quadrupolar mode of the enzyme distribution $l=2$ is unstable in the interval $-4.2<\mathrm{Pe}<-3.6$ while it predicts that all the modes are stable for $\mathrm{Pe}<-4.2$.

Conclusions. Our results identify the key parameters that govern the active self-polarization of the particle. As shown by Eq. (8) and by the definition of Pe, active self-polarization is favored by either decreasing the critical Péclet number, i.e., with intermediate enzyme coverage and slightly attractive enzyme-enzyme interactions, or by increasing Pe. The latter can be achieved in experiments by choosing bulky enzymes leading to a larger $f$, strong product-surface interactions leading to a large phoretic coefficient $b$, large catalytic rates $k_{\text {cat }}$ [46], large particles, and small enzyme mobility. If polarization takes place, then the velocity grows with the magnitude of Pe.

There is some similarity between the spontaneous polarization under nonequilibrium conditions and the classic equilibrium liquid-liquid demixing [57]. For instance, Fig. 4 shows that for small and large $\Gamma_{0}^{*}$ there is a region where a homogeneous solution coexists with a polarized one. In these regions, polarization occurs with a finite jump of the concentration dipole (and particle velocity), which is reminiscent of a first order phase transition. This analogy suggests that the phoretic flows act as an effective nonequilibrium attractive interaction. In the rest of the phase diagram, the transition to polarized states occurs with a continuous increase of the concentration dipole, which resembles a second order phase transition. However, since the mechanism that we discuss here is out-of-equilibrium requiring energy input from chemical reactions, the comparison with liquid-liquid demixing is only an analogy.

In summary, we have identified a mechanism for the self-propulsion of chemically active particles, which rather than having hard-wired asymmetry, spontaneously develop active polarization enabled by the lateral mobility of enzymes on their surface. In short, a perturbation of surface enzyme density in an otherwise uniformly coated particle results in an asymmetric reaction rate, which generates a gradient of product and phoretic flows along the particle surface. The advection of mobile enzymes over the surface by these flows reinforces the initial perturbation, ultimately leading to a self-sustained polarization of the enzyme distribution and steady particle motion. This mechanism bears similarity to other active mechanochemical symmetry-breaking instabilities exploited by cells to divide, polarize or migrate [19-22,58]. Our results could be useful to design bio-mimicking active particles with an adaptive or controllable propulsion mechanism, which can be dynamically (dis-)engaged by sensing or tuning any of the physical parameters involved in the self-polarization instability, as mapped in the present study.

Acknowledgments. M.D.C. acknowledges funding from the European Union's Horizon 2020 research and innovation program under the Marie Skłodowska-Curie action (GA 712754), the Severo Ochoa programme (SEV-2014-0425), and the CERCA Programme/Generalitat de Catalunya. I.P. acknowledges support from MINECO/FEDER Project No. PGC2018-098373-B- 
100/FEDER-EU, DURSI Project No. 2017SGR-884, SNF Project No. 200021-175719, and the EU Horizon 2020 program through 766972-FET-OPENNANOPHLOW. M.A. acknowledges the support from the Generalitat de Catalunya (ICREA Academia Award No. 2017-SGR-1278), from the Spanish Ministry of Economy and Competitiveness, through the Severo Ochoa Programme (CEX2018-000797-S), and from the European Research Council (CoG-681434). S.S. acknowledges the BBVA Foundation for the MEDIROBOTS project, the CERCA program by the Generalitat de Catalunya, and support from Pencheng Scholarship of Shenzhen City in China.

[1] W. F. Paxton, K. C. Kistler, C. C. Olmeda, A. Sen, S. K. St. Angelo, Y. Cao, T. E. Mallouk, P. E. Lammert, and V. H. Crespi, Catalytic nanomotors: Autonomous movement of striped nanorods, J. Am. Chem. Soc. 126, 13424 (2004).

[2] J. R. Howse, R. A. L. Jones, A. J. Ryan, T. Gough, R. Vafabakhsh, and R. Golestanian, Self-Motile Colloidal Particles: From Directed Propulsion to Random Walk, Phys. Rev. Lett. 99, 048102 (2007).

[3] C. Bechinger, R. Di Leonardo, H. Löwen, C. Reichhardt, G. Volpe, and G. Volpe, Active particles in complex and crowded environments, Rev. Mod. Phys. 88, 045006 (2016).

[4] A. C. Hortelão, R. Carrascosa, N. Murillo-Cremaes, T. Patiño, and S. Sánchez, Targeting 3d bladder cancer spheroids with urease-powered nanomotors, ACS Nano 13, 429 (2018).

[5] S. Tang, F. Zhang, H. Gong, F. Wei, J. Zhuang, E. Karshalev, B. E.-F. de Ávila et al., Enzyme-powered janus platelet cell robots for active and targeted drug delivery, Sci. Robot. 5, eaba6137 (2020).

[6] A. C. Hortelao, C. Simo, M. Guix, S. Guallar-Garrido, E. Julian, D. Vilela, L. Rejc, P. Ramos-Cabrer, U. Cossio, V. Gomez-Vallejo et al., Monitoring the collective behavior of enzymatic nanomotors in vitro and in vivo by pet-ct, bioRxiv (2020).

[7] J. Parmar, D. Vilela, K. Villa, J. Wang, and S. Sánchez, Micro-and nanomotors as active environmental microcleaners and sensors, J. Am. Chem. Soc. 140, 9317 (2018).

[8] N. Sharifi-Mood, J. Koplik, and C. Maldarelli, Diffusiophoretic self-propulsion of colloids driven by a surface reaction: The sub-micron particle regime for exponential and van der waals interactions, Phys. Fluids 25, 012001 (2013).

[9] A. P. Bregulla and F. Cichos, Flow fields around pinned self-thermophoretic microswimmers under confinement, J. Chem. Phys. 151, 044706 (2019).

[10] J. de Graaf and S. Samin, Self-thermoelectrophoresis at low salinity, Soft Matter 15, 7219 (2019).

[11] S. Eloul, W. C. K. Poon, O. Farago, and D. Frenkel, Reactive Momentum Transfer Contributes to the Self-Propulsion of Janus Particles, Phys. Rev. Lett. 124, 188001 (2020).

[12] M. De Corato, X. Arqué, T. Patiño, M. Arroyo, S. Sánchez, and I. Pagonabarraga, Self-Propulsion of Active Colloids via Ion Release: Theory and Experiments, Phys. Rev. Lett. 124, 108001 (2020).

[13] I. Buttinoni, G. Volpe, F. Kümmel, G. Volpe, and C. Bechinger, Active brownian motion tunable by light, J. Phys.: Condens. Matter 24, 284129 (2012).

[14] S. Michelin and E. Lauga, Autophoretic locomotion from geometric asymmetry, Eur. Phys. J. E 38, 7 (2015).

[15] S. Michelin and E. Lauga, Geometric tuning of self-propulsion for janus catalytic particles, Sci. Rep. 7, 42264 (2017).

[16] A. Varma, T. D. Montenegro-Johnson, and S. Michelin, Clustering-induced self-propulsion of isotropic autophoretic particles, Soft Matter 14, 7155 (2018).

[17] R. D. Baker, T. Montenegro-Johnson, A. D. Sediako, M. J. Thomson, A. Sen, E. Lauga, and I. S. Aranson, Shape-programmed $3 \mathrm{~d}$ printed swimming microtori for the transport of passive and active agents, Nat. Commun. 10, 4932 (2019).

[18] R. Golestanian, T. B. Liverpool, and A. Ajdari, Propulsion of a Molecular Machine by Asymmetric Distribution of Reaction Products, Phy. Rev. Lett. 94, 220801 (2005).

[19] V. Ruprecht, S. Wieser, A. Callan-Jones, M. Smutny, H. Morita, K. Sako, V. Barone, M. Ritsch-Marte, M. 
Sixt, R. Voituriez, and C.-P. Heisenberg, Cortical contractility triggers a stochastic switch to fast amoeboid cell motility, Cell 160, 673 (2015).

[20] M. Bergert, A. Erzberger, R. A. Desai, I. M. Aspalter, A. C. Oates, G. Charras, G. Salbreux, and E. K. Paluch, Force transmission during adhesion-independent migration, Nat. Cell Biol. 17, 524 (2015).

[21] A. C. Callan-Jones, V. Ruprecht, S. Wieser, C. P. Heisenberg, and R. Voituriez, Cortical Flow-Driven Shapes of Nonadherent Cells, Phys. Rev. Lett. 116, 028102 (2016).

[22] A. Farutin, J. Étienne, C. Misbah, and P. Recho, Crawling in a Fluid, Phys. Rev. Lett. 123, 118101 (2019).

[23] T. Patiño, X. Arqué, R. Mestre, L. Palacios, and S. Sánchez, Fundamental aspects of enzyme-powered micro-and nanoswimmers, Acc. Chem. Res. 51, 2662 (2018).

[24] T. Patiño, N. Feiner-Gracia, X. Arqué, A. Miguel-López, A. Jannasch, T. Stumpp, E. Schäffer, L. Albertazzi, and S. Sánchez, Influence of enzyme quantity and distribution on the self-propulsion of non-janus urease-powered micromotors, J. Am. Chem. Soc. 140, 7896 (2018).

[25] S. Ghosh, F. Mohajerani, S. Son, D. Velegol, P. J. Butler, and A. Sen, Motility of enzyme-powered vesicles, Nano Lett. 19, 6019 (2019).

[26] A. Somasundar, S. Ghosh, F. Mohajerani, L. N. Massenburg, T. Yang, P. S. Cremer, D. Velegol, and A. Sen, Positive and negative chemotaxis of enzyme-coated liposome motors, Nat. Nanotech. 14, 1129 (2019).

[27] X. Arqué, A. Romero-Rivera, F. Feixas, T. Patiño, S. Osuna, and S. Sánchez, Intrinsic enzymatic properties modulate the self-propulsion of micromotors, Nat. Commun. 10, 2826 (2019).

[28] S. Michelin, E. Lauga, and D. Bartolo, Spontaneous autophoretic motion of isotropic particles, Phys. Fluids 25, 061701 (2013).

[29] M. Schmitt and H. Stark, Swimming active droplet: A theoretical analysis, Europhys. Lett. 101, 44008 (2013).

[30] P. de Buyl, A. S. Mikhailov, and R. Kapral, Self-propulsion through symmetry breaking, Europhys. Lett. 103, 60009 (2013).

[31] Z. Izri, M. N. van der Linden, S. Michelin, and O. Dauchot, Self-Propulsion of Pure Water Droplets by Spontaneous Marangoni-Stress-Driven Motion, Phys. Rev. Lett. 113, 248302 (2014).

[32] C. C. Maass, C. Krüger, S. Herminghaus, and C. Bahr, Swimming droplets, Annu. Rev. Condens. Matter Phys. 7, 171 (2016).

[33] S. Michelin, S. Game, E. Lauga, E. Keaveny, and D. Papageorgiou, Spontaneous onset of convection in a uniform phoretic channel, Soft Matter 16, 1259 (2020).

[34] M. Tanaka, J. Hermann, I. Haase, M. Fischer, and S. G. Boxer, Frictional drag and electrical manipulation of recombinant proteins in polymer-supported membranes, Langmuir 23, 5638 (2007).

[35] A. Goychuk and E. Frey, Protein Recruitment Through Indirect Mechanochemical Interactions, Phys. Rev. Lett. 123, 178101 (2019).

[36] C. Tozzi, N. Walani, and M. Arroyo, Out-of-equilibrium mechanochemistry and self-organization of fluid membranes interacting with curved proteins, New J. Phys. 21, 093004 (2019).

[37] S. Gupta, K. K. Sreeja, and S. Thakur, Autonomous movement of a chemically powered vesicle, Phys. Rev. E 92, 042703 (2015).

[38] J. L. Moran and J. D. Posner, Phoretic self-propulsion, Annu. Rev. Fluid Mech. 49, 511 (2017).

[39] J. L. Anderson, Colloid transport by interfacial forces, Annu. Rev. Fluid Mech. 21, 61 (1989).

[40] K. Jacobson, A. Ishihara, and R. Inman, Lateral diffusion of proteins in membranes, Annu. Rev. Physiol. 49, 163 (1987).

[41] M. L Huggins, Solutions of long chain compounds, J. Chem. Phys. 9, 440 (1941).

[42] P. J. Flory, Thermodynamics of high polymer solutions, J. Chem. Phys. 10, 51 (1942).

[43] T. Baumgart, A. T. Hammond, P. Sengupta, S. T. Hess, D. A. Holowka, B. A. Baird, and W. W. Webb, Large-scale fluid/fluid phase separation of proteins and lipids in giant plasma membrane vesicles, Proc. Natl. Acad. Sci. USA 104, 3165 (2007).

[44] J. Carr, M. E. Gurtin, and M. Slemrod, Self-propulsion of active colloids via ion release: Theory and experiments, Arch. Rational Mech. Anal. 86, 317 (1984).

[45] S.-K. Hu, L.-T. Huang, and L. Chao, Membrane species mobility under in-lipid-membrane forced convection, Soft Matter 12, 6954 (2016). 
[46] See Supplemental Material at http://link.aps.org/supplemental/10.1103/PhysRevFluids.5.122001 for additional information on the dimensionless equations, the linear stability analysis, the numerical method used for the nonlinear simulations, and the relevant physico-chemical parameters of enzymes used in previous experimental studies.

[47] M. J. Lighthill, On the squirming motion of nearly spherical deformable bodies through liquids at very small reynolds numbers, Commun. Pure Appl. Mat. 5, 109 (1952).

[48] J. R. Blake, A spherical envelope approach to ciliary propulsion, J. Fluid Mech. 46, 199 (1971).

[49] E. Lauga and S. Michelin, Stresslets Induced by Active Swimmers, Phys. Rev. Lett. 117, 148001 (2016).

[50] J. Agudo-Canalejo and R. Golestanian, Active Phase Separation in Mixtures of Chemically Interacting Particles, Phys. Rev. Lett. 123, 018101 (2019).

[51] B. Liebchen and H. Löwen, Which interactions dominate in active colloids? J. Chem. Phys. 150, 061102 (2019).

[52] S. Saha, S. Ramaswamy, and R. Golestanian, Pairing, waltzing and scattering of chemotactic active colloids, New J. Phys. 21, 063006 (2019).

[53] R. Singh, R. Adhikari, and M. E. Cates, Competing chemical and hydrodynamic interactions in autophoretic colloidal suspensions, J. Chem. Phys. 151, 044901 (2019).

[54] E. Kanso and S. Michelin, Phoretic and hydrodynamic interactions of weakly confined autophoretic particles, J. Chem. Phys. 150, 044902 (2019).

[55] B. Nasouri and R. Golestanian, Exact Phoretic Interaction of Two Chemically Active Particles, Phys. Rev. Lett. 124, 168003 (2020).

[56] A. Scagliarini and I. Pagonabarraga, Unravelling the role of phoretic and hydrodynamic interactions in active colloidal suspensions, Soft Matter 16, 8893 (2020).

[57] R. A. L. Jones, Soft Condensed Matter, Vol. 6 (Oxford University Press, Oxford, 2002).

[58] A. Mietke, V. Jemseena, K. V. Kumar, I. F. Sbalzarini, and F. Jülicher, Minimal Model of Cellular Symmetry Breaking, Phys. Rev. Lett. 123, 188101 (2019). 